\title{
Optically Tunable Metamaterial Perfect Absorber on Highly Flexible Substrate ${ }^{\dagger}$
}

\section{Authors:}

Xiaoguang Zhao ${ }^{\text {a }}$, Kebin Fan ${ }^{\text {a }}$, Jingdi Zhang ${ }^{b}$, Huseyin R. Seren ${ }^{\text {a }}$, Grace D. Metcalfe ${ }^{c}$, Micheal Wraback ${ }^{c}$, Richard D. Averitt ${ }^{b, d}$ and Xin Zhang ${ }^{a *}$

\section{Affiliations:}

${ }^{a}$ Department of Mechanical Engineering, Boston University, Boston, MA 02215 USA

${ }^{\mathrm{b}}$ Department of Physics, Boston University, Boston, MA 02215 USA

${ }^{\mathrm{c}}$ U.S. Army Research Laboratory, Adelphi, MD 20783 USA

${ }^{\mathrm{d}}$ Department of Physics, University of California, San Diego, La Jolla, California 92093 USA

\section{* Corresponding author:}

Xin Zhang, $\mathrm{PhD}$

Department of Mechanical Engineering

Boston University

110 Cummington Mall

Boston, Massachusetts 02215

(617) $358-2702$

xinz@bu.edu

\footnotetext{
${ }^{\dagger}$ This paper was presented at the $27^{\text {th }}$ IEEE MEMS conference, held in San Francisco, CA, USA, Jan. 26-30, 2014 [1], and is an expansion of paper in the Technical Digest of this meeting.
} 


\section{ABSTRACT}

We present our recent progress on a highly flexible tunable perfect absorber at terahertz frequencies. Metamaterial unit cells were patterned on thin GaAs patches, which were fashioned in an array on a $10 \mu \mathrm{m}$ polyimide substrate via semiconductor transfer technique, and the backside of the substrate was coated with gold film as a ground plane. Optical-pump THz-probe reflection measurements show that the absorptivity can be tuned up to $25 \%$ at $0.78 \mathrm{THz}$ and $40 \%$ at $1.75 \mathrm{THz}$ through photo-excitation of free carriers in GaAs layers in presence of 800nm pump beam. Our flexible tunable MM perfect absorber has potential applications in energy harvesting, THz modulation and even camouflages coating.

Keywords: Metamaterials perfect absorber, Optically Tuning, Flexible, Terahertz

\section{INTRODUCTION}

During the past decade, electromagnetic (EM) metamaterials (MMs) have attracted considerable interest due to their unusual EM response and promising applications [2]. MM perfect absorbers (MPAs) have been considered as one particular and important branch of MMs enabling near-unity absorption in a thin slab with thickness much smaller than $\lambda / 4[2,3]$. Since 2008, a great number of examples of MPAs have been demonstrated in different frequency regimes, including microwave [4], terahertz $(\mathrm{THz})$ [5], infrared [6], mid-infrared [7], nearinfrared [8] and visible frequency [9].

Tunable metamaterials have shown the ability to manipulate EM waves to build modulators, sensors, detectors, and many other devices [10]. Routes to realize tunable MM include photoexcitation [11], electrical [12, 13], phase change [14], and mechanical reconfiguration [15]. In our previous work [16], we demonstrated the fabrication and characterization of optically tunable metamaterials on a thin and flexible substrate. The gold MM structure on GaAs patches were successfully transferred to $4 \mu \mathrm{m}$ thick polyimide and we achieved $60 \%$ modulation depth at 
$0.98 \mathrm{THz}$ with the pump beam power of $8 \mathrm{~mW}$.

In the same vein, introducing dynamics into MPAs provides a new path towards exotic devices thus extending applications of MPAs for imaging, sensing, energy harvesting [17], spatial light modulators [18], and even camouflage of IR emission from detection [19]. Furthermore, the integration of tunable MPAs on ultrathin and flexible substrates enables high adaptability to wrap and fit on arbitrary surfaces for a range of practical applications.

Here, we report our progress on a novel flexible tunable perfect absorber in the THz regime. We used the transfer printing method to pattern the MMs and GaAs patches on polyimide and coated the backside with gold thin film to construct the flexible tunable perfect absorber. Subsequently, the device was characterized by optical pump THz-probe spectroscopy. The modulation depth in absorptivity was measured as $25 \%$ at $0.78 \mathrm{THz}$ and $40 \%$ at $1.75 \mathrm{THz}$ when the pump beam power was $25.6 \mathrm{~mW}$ with original absorptivity of $84 \%$ and $99 \%$, respectively. Our flexible perfect absorber may find applications in a variety of areas including camouflage coating, THz modulation and switching, energy harvesting, and chemical/biological sensors.

\section{DESIGN AND FABRICATION}

Our absorber makes use of photo-excitation of carriers in GaAs to dynamically control the response of electric split-ring resonators (eSRRs) overlying the GaAs patches. Fig. 1 (a) schematically shows the tunable flexible perfect absorber photo-excited by an $800 \mathrm{~nm}$ optical pump beam. The unit cell of the absorber as shown in Fig. 1 (b) is composed of GaAs patch, a gold eSRR, polyimide spacer and gold ground plane. The array of GaAs patches and eSRRs composes the tunable MM, sandwiching the polyimide spacer along with the ground plane. The windows in each unit are intentionally fabricated to etch the sacrificial layer during the releasing of the flexible absorber.

The metallic eSRRs and ground plane together determine the effective electromagnetic 
properties (i.e. permittivity- $\varepsilon_{e f f}$ and permeability- $\mu_{e f f}$ ) of the perfect absorber. When the electric field of the incident THz wave is perpendicular to the capacitive gap in the eSRR structure, it will couple to the eSRR and excite the LC resonance that arise an effective permittivity. At the same time, the magnetic field generates circulating currents between the two metallic layers as shown in Fig. 2 (a) and (b), resulting in an effective permeability. According to Fresnel's law, the reflectivity of a transverse magnetic (TM) polarized wave from the interface of air/MPA is

$$
R_{T M}=\left|r_{T M}\right|^{2}=\left|\frac{Z_{M} \sqrt{\left(\mu_{e f f} Z_{0}\right)^{2}-Z_{M}^{2} \sin ^{2} \theta}-\left(\mu_{e f f} Z_{0}^{2}\right) \cos \theta}{Z_{M} \sqrt{\left(\mu_{e f f} Z_{0}\right)^{2}-Z_{M}^{2} \sin ^{2} \theta}+\left(\mu_{e f f} Z_{0}^{2}\right) \cos \theta}\right|^{2}
$$

where $\mathrm{Z}_{M}=\sqrt{ }\left[\left(\mu_{0} \cdot \mu_{\text {eff }}\right) /\left(\varepsilon_{0} \cdot \varepsilon_{e f f}\right)\right]$ is the effective impedance of the metamaterial perfect absorber, $Z_{0}$ $=\sqrt{ }\left(\mu_{0} / \varepsilon_{0}\right)$ is the impedance of free space, and $\theta$ is the incident angle. For the normal incident condition, the reflectivity can be simplified as

$$
R_{T M}=\left|r_{T M}\right|^{2}=\left|\left(Z_{M}-Z_{0}\right) /\left(Z_{M}+Z_{0}\right)\right|
$$

With an appropriate geometric design, the impedance of the MPA can be matched with freespace around the resonant frequencies to eliminate the reflection; meanwhile, the ground plane ensures that the transmission $(T)$ is negligibly small. The absorptivity can be calculated by

$$
A=1-R_{T M}-T \approx 1-R_{T M}
$$

Fig. 2 (c) shows the near-unity absorption achieved at the LC resonance of a device optimized for normal incidence without transmission and reflection. Although the absorption degrades for oblique angles, it is possible to optimize the device for a particular angle by simply increasing the dielectric spacer thickness.

MPAs provide a new approach to construct absorbers, in which the permittivity and permeability can be engineered independently. That is, the absorption response can be easily tailored by modifying either the permittivity or permeability. Simply changing the capacitance in 
the eSRRs' gap can modify the resonant frequency, amplitude and phase of the reflection, thus altering the effective permittivity, eeff. If an optical pump beam is incident on our MM perfect absorber (as shown in Fig. 1), the free carriers will be generated in the GaAs patches due to photo-excitation, which can in turn lead to an increase their conductivity. This will alter the $\varepsilon_{e f f}$ and cause a mismatch of impedance and higher reflection. Consequently, the absorption can be modulated in this fashion.

Fig. 3 shows the fabrication process of the flexible perfect absorber. Initially, a 300nm sacrificial Al $0.95 \mathrm{Ga} 0.05 \mathrm{As}$ layer and a 300nm semi-insulating (SI) GaAs layer were epitaxially grown on a 2" SI-GaAs wafer. Then, a $15 \times 15 \mathrm{~mm}^{2}$ array of $200 \mathrm{~nm}$ thick gold eSRRs was patterned on the epitaxial SI-GaAs layer using the lift-off process [Fig. 3 (a)]. The epilayer was wet etched with citric acid: $\mathrm{H}_{2} \mathrm{O}_{2}$ (10:1) solution to form the GaAs patches that are concentric with the eSRRs after photoresist was spun on and patterned [Fig. 3 (b)]. The next step was to dip the wafer into diluted HF solution to remove the uncovered sacrificial layer [Fig. 3 (c)]. The sacrificial layer was then etched in isotropic manner, yielding an undercut about 500nm underneath GaAs layer. Subsequently, a 10 $\mu$ m thick polyimide (PI5878G, HD Microsystems) layer was spun on and cured at $275^{\circ} \mathrm{C}$ [Fig. 3 (d)]. The undercut volume was filled with polyimide. Next, the polyimide was etched by RIE with oxygen gas to define the etching windows, following with wet etching of GaAs and soaking the wafer in diluted HF solution [Fig. 3 (e)]. After the entirety of the sacrificial layer was etched, the polyimide was released from the substrate and the GaAs patches and eSRR were successfully transferred to the polyimide. Finally, the backside of the released polyimide was coated with a $200 \mathrm{~nm}$ thick gold film via ebeam evaporation to form the ground plane [Fig. 3 (f)]. It should be emphasized that this fabrication process can be applied to other semiconductors (e.g. silicon and InAs) under which 
the sacrificial layer can be grown.

Fig. 4 (a) and (b) demonstrate the eSRR array of the flexible perfect absorber and the dimensions of the fabricated eSRR unit, respectively. The periodicity $(\mathrm{P})$ of the unit cells is $57 \mu \mathrm{m}$. The side-width of the GaAs patch and SRR are $54 \mu \mathrm{m}$ and $50 \mu \mathrm{m}$, respectively. The linewidth and gap in the SRR structure are $4 \mu \mathrm{m}$. The fabricated perfect absorber is highly flexible, as demonstrates by the capacity to wrap this device on a plastic vial as in Fig. 4 (c).

\section{EXPERIMENT AND RESULTS}

The reflection response of the MPA was characterized by optical pump THz-probe (OPTP) spectroscopy where a broadband $\mathrm{THz}$ pulse is created in a $\mathrm{ZnTe}$ crystal via optical rectification [16]. The experiment was conducted in a humidity-controlled environment where the humidity was less than $0.1 \%$ at room temperature. The flexible absorber sample was mounted on a holder so that the transverse magnetic (TM) $\mathrm{THz}$ pulses were $45^{\circ}$ incidence to the sample, as well as a gold coated reflector that is used as the reference. The OPTP spectroscopy system delivered amplified $35 \mathrm{fs}$ laser pulses at center wavelength of $800 \mathrm{~nm}$ and a repetition rate of $1 \mathrm{kHz}$ to excite the free carriers in the GaAs patches. As the lifetime of the photo-excited carrier in GaAs is about $1 \mathrm{~ns}[15]$, the THz probe pulse was fixed to arrive on the sample $10 \mathrm{ps}$ after the $800 \mathrm{~nm}$ pump pulse to ensure a steady accumulation of the carriers was established.

The experimental results of the reflection spectrum at different pump power are shown in Fig. 5 (a). In the absence of the 800nm pump beam, two resonant modes coupled to the $\mathrm{THz}$ field. The lower frequency mode corresponded to $0.78 \mathrm{THz}$ with the reflection amplitude of $40 \%$ while the higher one is at $1.75 \mathrm{THz}$ with the reflection amplitude of $8 \%$. The transmission of the THz field through the MPA was also measured. Due to the existence of the ground plane, the maximum amplitude of transmission coefficient was approximately 5\%, corresponding to $0.25 \%$ 
transmissivity (T), which was negligible. The absorptivity was estimated by Eq (3) and plotted in Fig.5 (b). The resonance strength of the two modes became weaker and absorptivity decreased when the pump power increased from $0 \mathrm{~mW}$ to $3.2 \mathrm{~mW}$. If the pump power was high enough, specifically, higher than $6.4 \mathrm{~mW}$ (equivalent to a fluence of $32 \mu \mathrm{J} / \mathrm{cm} 2$ ), the lower mode was completely damped since the pump beam served to excite sufficient photo-carriers in the GaAs; at the same time, the higher resonance frequency was seen to redshift with the increase in the pump power. The quality factors at each mode became small and a broadband absorber was achieved in the medium pump power level. When the pump power was high enough, a relatively high quality factor resonant absorption was observed at $0.95 \mathrm{THz}$ due to the redshift of the higher resonance mode in the absorptivity response. The reflectivity was tuned $25 \%$ at the lower resonance frequency and $40 \%$ at the higher frequency with $25.6 \mathrm{~mW}$ pump power.

\section{SIMULATION AND DISCUSSION}

To understand the nature of the modulation of the absorptivity, we performed the finitedifference simulation using CST Microwave Studio. The dimensions of the perfect absorber in our model were defined based on the fabricated samples. The gold layer was modeled as lossy metal with conductivity of $\sigma_{\text {gold }}=4.5 \times 10^{5}(\Omega \cdot \mathrm{cm})^{-1}$, while the $5 \mu \mathrm{m}$ polyimide was modeled as lossy dielectric material with dielectric constant of 2.88 and a tangent delta of 0.03 [15]. The 300nm thick semi-insulating (SI) GaAs layer was modeled as material with tunable conductivity, thereby modeling the photo-excited carrier density in the presence of pump power. The unit cell boundary condition was applied in our simulation and the $\mathrm{THz}$ source was obliquely incident on the sample at $45^{\circ}$.

The simulated results of modulation depth of absorptivity as a function of conductivity of SI-GaAs layer $\left(\sigma_{\mathrm{GaAs}}\right)$ are shown in Fig. 6 . With the conductivity increasing from $\sigma_{\mathrm{GaAs}}=1 \mathrm{~S} / \mathrm{cm}$ 
to $150 \mathrm{~S} / \mathrm{cm}$, the variation in simulated absorptivity demonstrates a similar trend to the experimental results [Fig. 5 (b)]. When the conductivity of GaAs is low, there are two resonance modes. The surface current distribution of the each mode is shown in Fig. 7 (a) and (b), respectively. The lower frequency mode corresponds to the LC mode in which the current circulates along the split ring resonator, while the higher frequency mode is the dipole mode in which current oscillates along the metallic line in the SRR. There is little current in the GaAs structure. With the increase of $\sigma_{\text {GaAs, }}$ the gap in the SRR will be shorted and the LC resonance is damped gradually. Meanwhile, the length of dipole resonator becomes longer, leading to the redshift of dipole resonance. When the conductivity of GaAs is at a medium level, for example, between $50-80 \mathrm{~S} / \mathrm{cm}$, the quality factor of the LC and dipole resonators are both low because of the high damping in the GaAs structure. Hence, broadband absorption can be realized in these conditions. When the conductivity of GaAs is high enough, the LC resonance will be eventually eliminated and only one resonance mode exists as shown in the red curve of Fig. 5. The surface current distribution at the resonance frequency when $\sigma_{\mathrm{GaAs}}$ is $150 \mathrm{~S} / \mathrm{cm}$ are shown in Fig. 7 (c), indicating that this mode is a dipole with longer length than Fig. 7 (b). Due to the increase of $\sigma_{\mathrm{GaAs}}$, the damping in the GaAs structure decreases and the quality factor increases.

The simulated results of tunable absorptivity agree well with the experimental results. This indicates that the modulation of absorptivity of our MPA structure originates from the change of conductivity due to photo-excitation. By controlling to conductivity of GaAs, we can switch our absorber among three working modes, (1) dual-band absorber, (2) broadband absorber and (3) single-band absorber.

We also calculated the power loss in the MPA device, illustrated in Fig. 8. The incident EM power can be dissipated in the GaAs, metallic SRR and polyimide. The dielectric loss in the 
GaAs and polyimide spacer [Fig. 8 (a) and (c)] exceeded the ohmic loss in metallic SRR [Fig. 8 (b)] at LC resonance when the conductivity of GaAs is $1 \mathrm{~S} / \mathrm{cm}$. The power loss in the center of the units is gap area of the SRR is dramatically large because the electric field across the gap is strong. However, when $\sigma_{\mathrm{GaAs}}$ is $150 \mathrm{~S} / \mathrm{cm}$, the capacitive gap of the LC resonator is shorted and the power loss around the gap drops greatly due to the weak electric field across the gap. In our absorber, most of the energy is dissipated in the GaAs patch. When conductivity of GaAs is low, the polyimide spacer contributes more to the absorption than the condition when GaAs is photoexcited.

\section{CONCLUSION}

A novel free-standing ultrathin tunable metamaterial perfect absorber was fabricated employing a semiconductor transfer technique and characterized by OPTP spectroscopy. The thickness of the perfect absorber is $10 \mu \mathrm{m}$, corresponding to $3 \%$ of the working wavelength at 1THz. The experimental results show that an $800 \mathrm{~nm}$ ultrafast laser beam with sufficient power on a GaAs patch can modulate the absorptivity by $25 \%$ at $0.78 \mathrm{THz}$ and $40 \%$ at $1.75 \mathrm{THz}$. The numerical simulation with varying GaAs conductivity was performed to understand the origin of the tunability and the results agreed well with the experiments. According to the simulation and experimental results, we can switch the MPA among dual-band absorber, broadband absorber and single-band absorber modes by controlling the conductivity of GaAs.

\section{ACKNOWLEDGEMENT}

This work was supported in part by the National Science Foundation under Contract ECCS 1309835, the Air Force Office of Scientific Research under Contract FA9550-09-1-0708, and DTRA under Contract W911NF-06-2-0040 administered by the Army Research Laboratory. The authors would like to thank Boston University Photonics Center for technical support. 


\section{REFERENCES}

[1] X. Zhao, K. Fan, J. Zhang, H.R. Seren, G.D. Metcalfe, M. Wraback, R.D. Averitt, X. Zhang, Design, fabrication and characterization of tunable perfect absorber on flexible substrate, Technical Digest of 27th IEEE International Conference on MEMS, San Francisco, CA, USA, 2014, 84-87.

[2] C.M. Watts, X. Liu, W.J. Padilla, Metamaterial electromagnetic waver absorbers, Advanced Material, 24 (2012) 98-120.

[3] L. Huang, H.T. Chen, A brief review on terahertz metamaterial perfect absorbers, Terahertz Science and Technology, 6 (2013) 26-39.

[4] N.I. Landy, S. Sajuyigbe, J.J. Mock, D.R. Smith, W.J. Padilla, Perfect metamaterial absorber, Physical Review Letters, 100 (2008) 207402.

[5] H. Tao, N.I. Landy, C.M. Bingham, X. Zhang, R.D. Averitt, W.J. Padilla, A metamaterial absorber for the terahertz regime: Design, fabrication and characterization, Optical Express, 16 (2008) 7181-7188.

[6] Y. Avitzour, Y.A. Urzhumov, G. Shvets, Wide-angle infrared absorber based on a negativeindex plasmonic metamaterial, Physical Review B, 79 (2009) 045131.

[6] X. Liu, T. Starr, A.F. Starr, W.J. Padilla, Infrared spatial and frequency selective metamaterial with near-unity absorbance, Physical Review Letter, 104 (2010) 208403

[7] J. Hao, J. Wang, X. Liu, W.J. Padilla, L. Zhou, M. Qiu, High performance optical absorber based on a plasmonic metamaterial, Applied Physics Letters, 96 (2010) 251104.

[8] K. Aydin, V.E. Ferry, R.M. Briggs, H.A. Atwater, Broadband polarization-independent resonant light absorption using ultrathin plasmonic super absorbers, Nature Communications, $2(2011) 517$. 
[10] N.I. Zheludev, Y.S. Kivshar, From metamaterials to metadevices, Nature Materials, 11 (2012) 917-924.

[11]K. Fan, A.C. Strikwerda, X. Zhang, R.D. Averitt, Three-dimensional broadband tunable terahertz metamaterials, Physical Review B, 87 (2013) 161104.

[12]H.T. Chen, W.J. Padilla, M.J. Cich, A.K. Azad, R.D. Averitt, and A.J. Taylor, A metamaterials solid-state terahertz phase modulator, Nature Photon, 3 (2009) 148-151.

[13]D. Shrekenhamer, W.C. Chen, W.J. Padilla, Liquid Crystal Tunable Metamaterial Perfect Absorber, Physical Review Letter, 110 (2013) 177403.

[14]T. Driscoll, S. Palit, M.M. Qazilbash, D.R. Smith, D.N. Basov, et al. Dynamic tuning of an infrared hybrid-metamaterial resonance using vanadium dioxide, Applied Physics Letter, 93 (2008) 024101.

[15]X. Liu, W. J. Padilla, Dynamic Manipulation of Infrared Radiation with MEMS Metamaterials, Advanced Optical Material, 1 (2013) 559-562.

[16]K. Fan, X. Zhao, J. Zhang, K. Geng, G.R. Keiser, H.R. Seren, G.D. Metcalfe, M. Wraback, X. Zhang, R.D. Averitt, Optically Tunable Terahertz Metamaterials on Highly Flexible Substrates, IEEE Transaction on Terahertz Science and Technology, 3 (2013) 702-708.

[17]D. Shrekenhamer, W.C. Chen, W.J. Padilla, Liquid crystal tunable metamaterial absorber, Physical Review Letters, 110 (2013) 177403.

[18]S. Savo, D. Shrekenhamer, W.J. Padilla, Liquid crystal metamaterial absorber spatial light modulator for THz applications, Advanced Optical Materials, 2 (2014) 275-279.

[19]X. Liu, T. Tyler, T. Starr, A.F. Starr, N.M. Jokerst, W.J. Padilla, Taming the blackbody with infrared metamaterials as selective thermal emitters, Physical Review Letters, 107 (2011) 045901. 


\section{Figure Captions}

Figure 1: (a) The illustration of flexible tunable MM perfect absorber illuminated by an 800nm beam; (b) unit cell of the MM perfect absorber (top view and cross section view).

Figure 2: (a) Simulated current distribution on the eSRR structure; (b) simulated current distribution on the ground plane; (c) simulated reflectivity (R: black curve), transmissivity ( $\mathrm{T}$ : green curve) and absorptivity (A: black curve) for the tunable MM perfect absorber.

Figure 3: Fabrication process of the flexible tunable MM perfect absorber. (a)Pattern ESRR structure with lift-off; (b) Wet etch the GaAs; (c) Wet etch $\mathrm{Al}_{0.95} \mathrm{Ga}_{0.05} \mathrm{As}$; (d) Spin on polyimide; (e) Open the releasing hole on polyimide and wet etch the GaAs and $\mathrm{Al}_{0.95} \mathrm{Ga}_{0.05} \mathrm{As}$ to release the whole structure; (f) Coat the backside of polyimide with gold thin film to form the ground plane.

Figure 4: Images of the perfect absorber after releasing. (a) Microscopic images of the eSRRs; (b) magnified view of one eSRR unit, $l_{i}=54 \mu \mathrm{m}, l=50 \mu \mathrm{m}, w=4 \mu \mathrm{m}, g=4 \mu \mathrm{m}, P=57 \mu \mathrm{m}$; (c) the flexible tunable MM perfect absorber wrapped on a plastic vial.

Figure 5: Experimental results: reflection coefficient (a) and absorptivity (b) at different pump powers.

Figure 6: Simulated absorptivity with different conductivity of GaAs.

Figure 7: Simulated surface current distribution at (a) $0.8 \mathrm{THz}$ when $\sigma_{\mathrm{GaAs}}=1 \mathrm{~S} / \mathrm{cm}$, (b) $1.8 \mathrm{THz}$ when $\sigma_{\mathrm{GaAs}}$ $=1 \mathrm{~S} / \mathrm{cm}$, and (c) $0.9 \mathrm{THz}$ when $\sigma_{\mathrm{GaAs}}=150 \mathrm{~S} / \mathrm{cm}$.

Figure 8: Simulated power loss at different slice across the MPA structure. (a) - (c) The power loss in different material when $\sigma \mathrm{GaAs}$ is $1 \mathrm{~S} / \mathrm{cm}$. (d) - (f) The power loss when $\sigma \mathrm{GaAs}$ is $150 \mathrm{~S} / \mathrm{cm}$. 


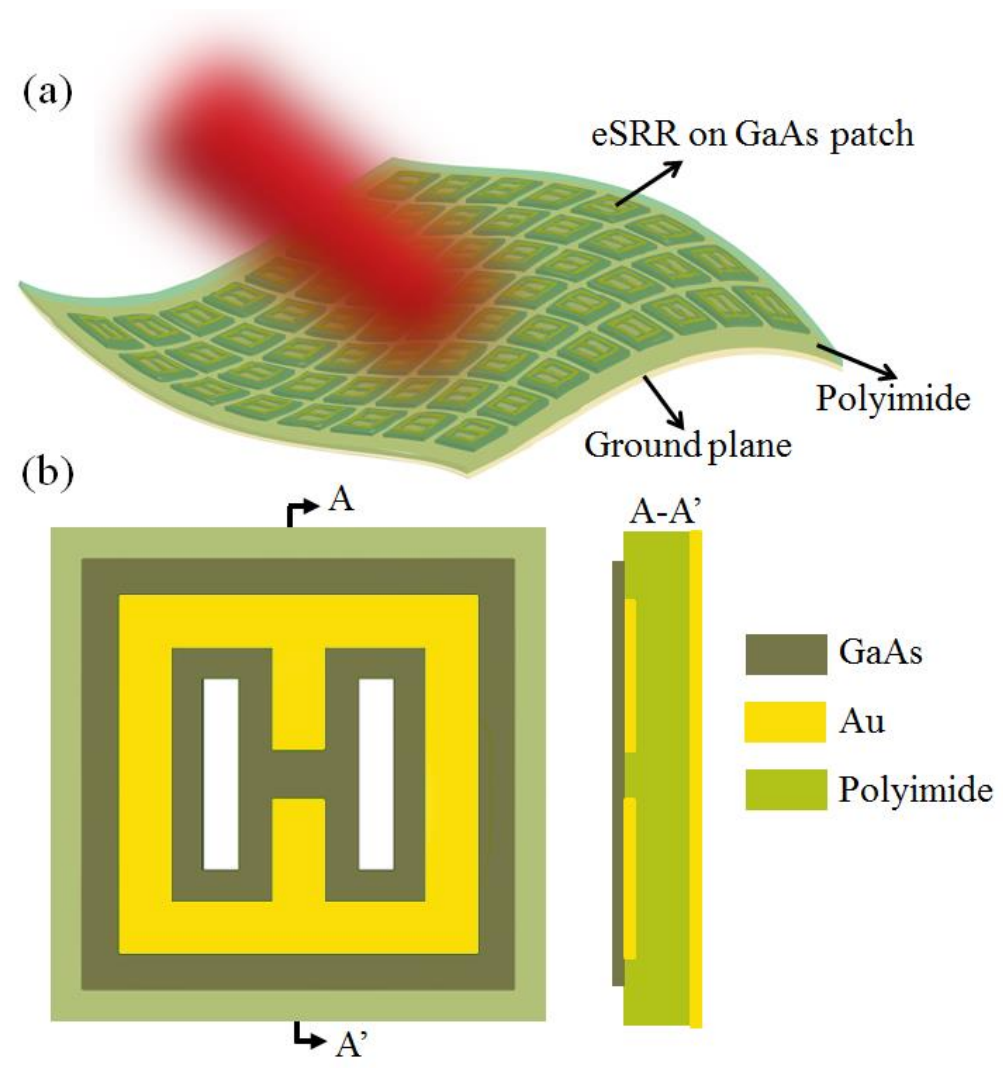

Figure 1: (a) The illustration of flexible tunable MM perfect absorber illuminated by an 800nm beam; (b) unit cell of the MM perfect absorber (top view and cross section view). 
(a)

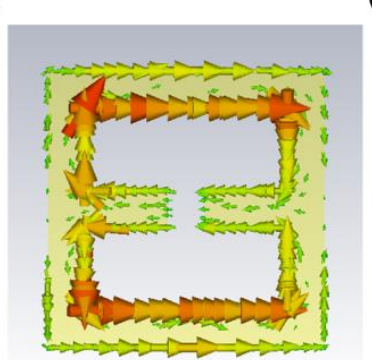

(c)

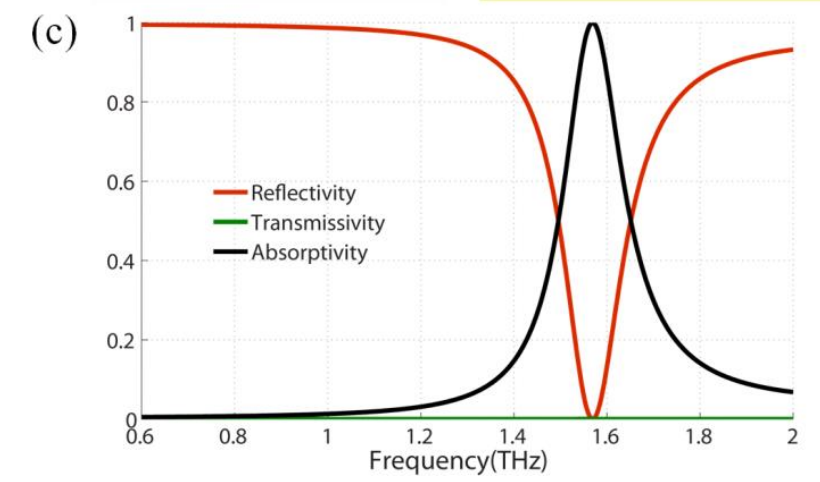

(b)

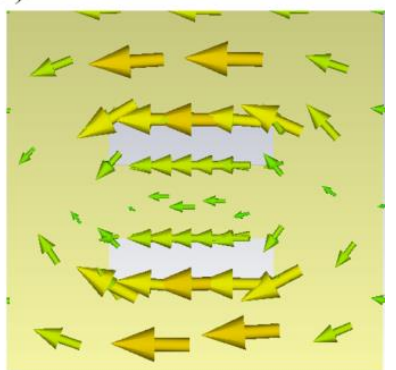

Figure 2: (a) Simulated current distribution on the eSRR structure; (b) simulated current distribution on the ground plane; (c) simulated reflectivity (R: black curve), transmissivity (T: green curve) and absorptivity (A: black curve) for the tunable MM perfect absorber. 

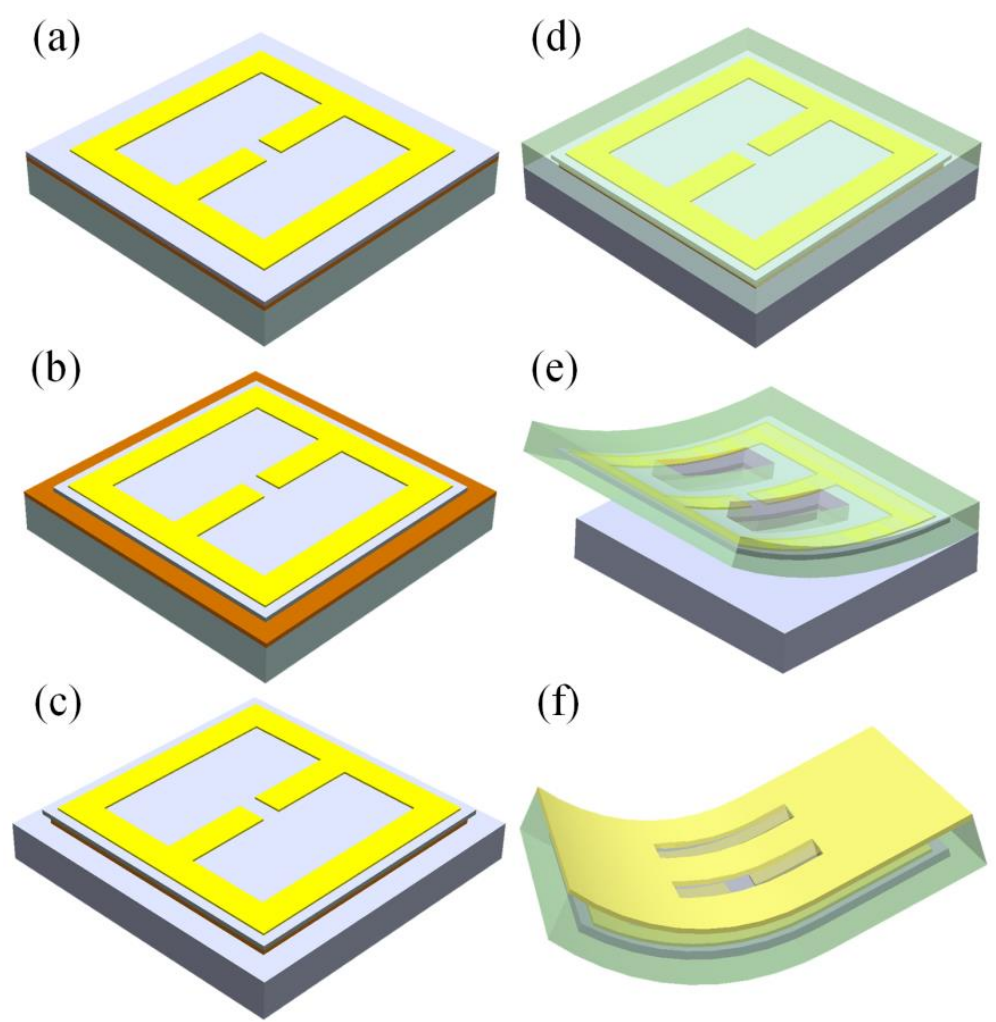

(f)

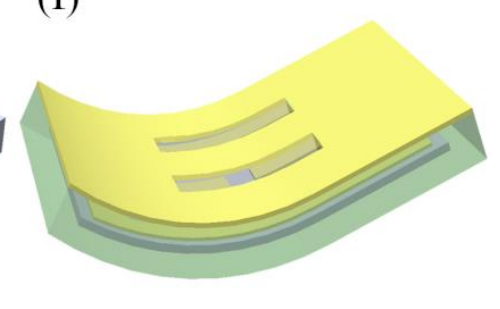

Figure 3: Fabrication process of the flexible tunable MM perfect absorber. (a)Pattern ESRR structure with lift-off; (b) Wet etch the GaAs; (c) Wet etch Al $0.95 \mathrm{Ga} 0.05 \mathrm{As}$; (d) Spin on polyimide; (e) Open the releasing hole on polyimide and wet etch the GaAs and $\mathrm{Al}_{0.95} \mathrm{Ga} 0.05 \mathrm{As}$ to release the whole structure; (f) Coat the backside of polyimide with gold thin film to form the ground plane. 

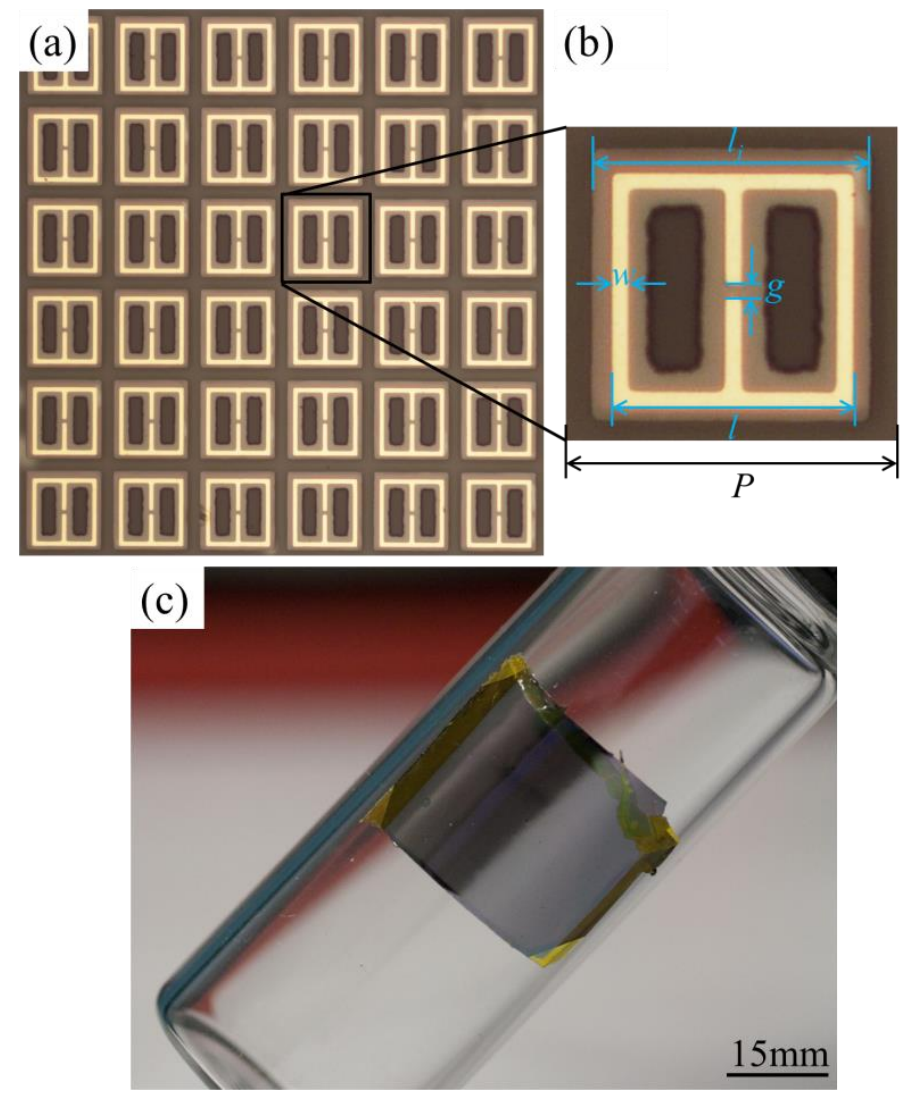

Figure 4: Images of the perfect absorber after releasing. (a) Microscopic images of the eSRRs; (b) magnified view of one eSRR unit, $l_{i}=54 \mu \mathrm{m}, l=50 \mu \mathrm{m}, w=4 \mu \mathrm{m}, g=4 \mu \mathrm{m}, P=57 \mu \mathrm{m}$; (c) the flexible tunable MM perfect absorber wrapped on a plastic vial. 

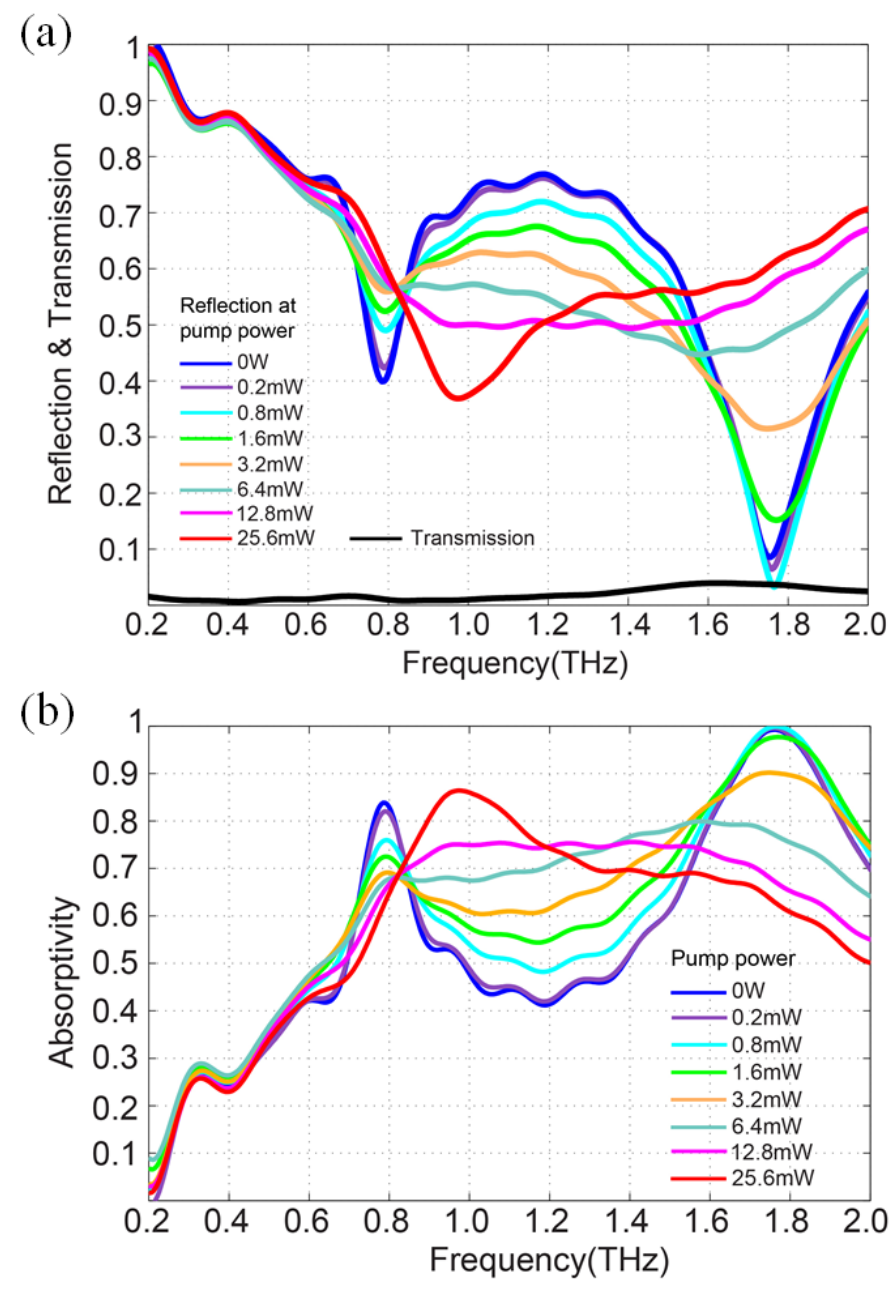

Figure 5: Experimental results: reflection coefficient (a) and absorptivity (b) at different pump powers. 


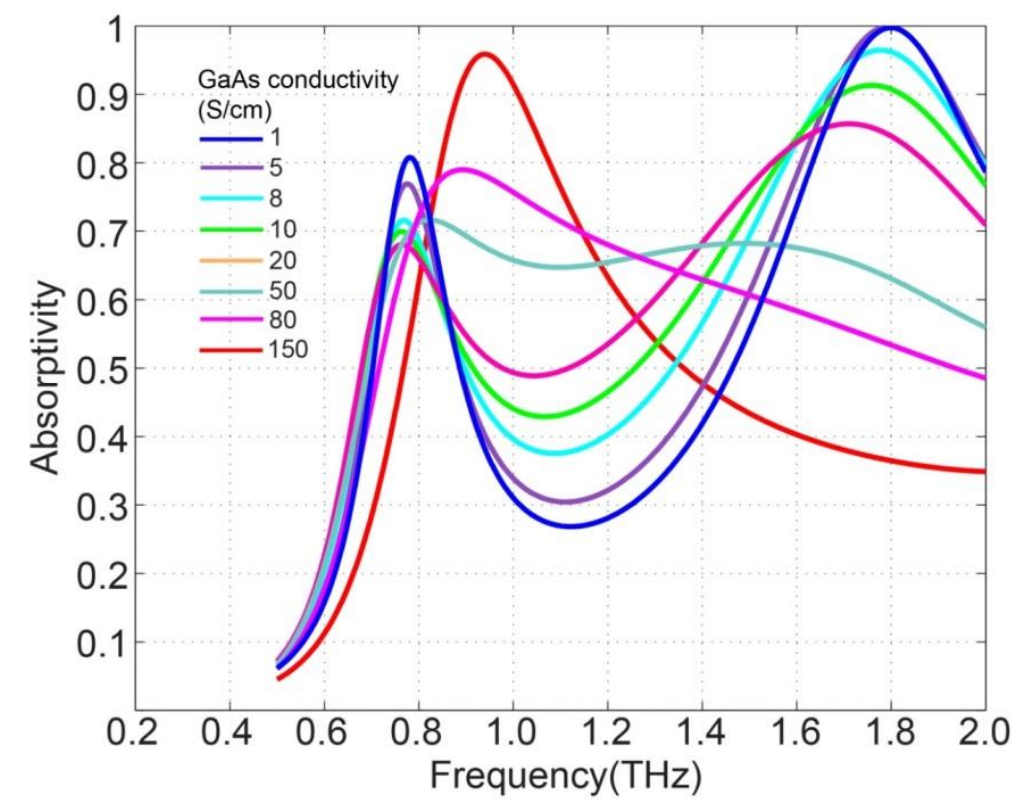

Figure 6: Simulated absorptivity with different conductivity of GaAs. 
(a)

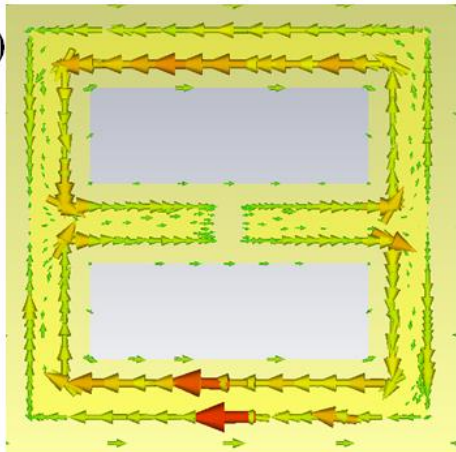

(b)

(c)

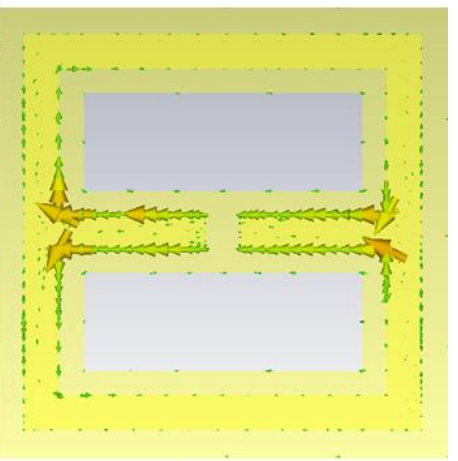

(c)

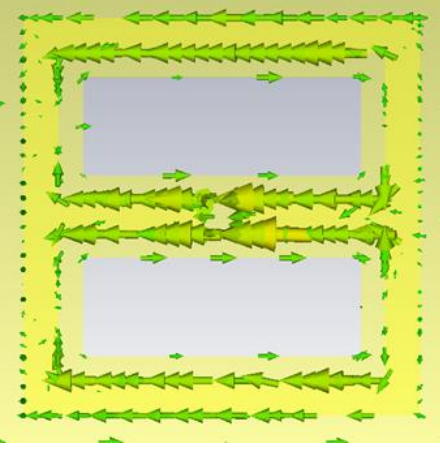

Figure 7: Simulated surface current distribution at (a) $0.8 \mathrm{THz}$ when $\sigma_{\mathrm{GaAs}}=1 \mathrm{~S} / \mathrm{cm}$, (b) $1.8 \mathrm{THz}$ when $\sigma_{\mathrm{GaAs}}=1 \mathrm{~S} / \mathrm{cm}$, and (c) $0.9 \mathrm{THz}$ when $\sigma_{\mathrm{GaAs}}=150 \mathrm{~S} / \mathrm{cm}$. 

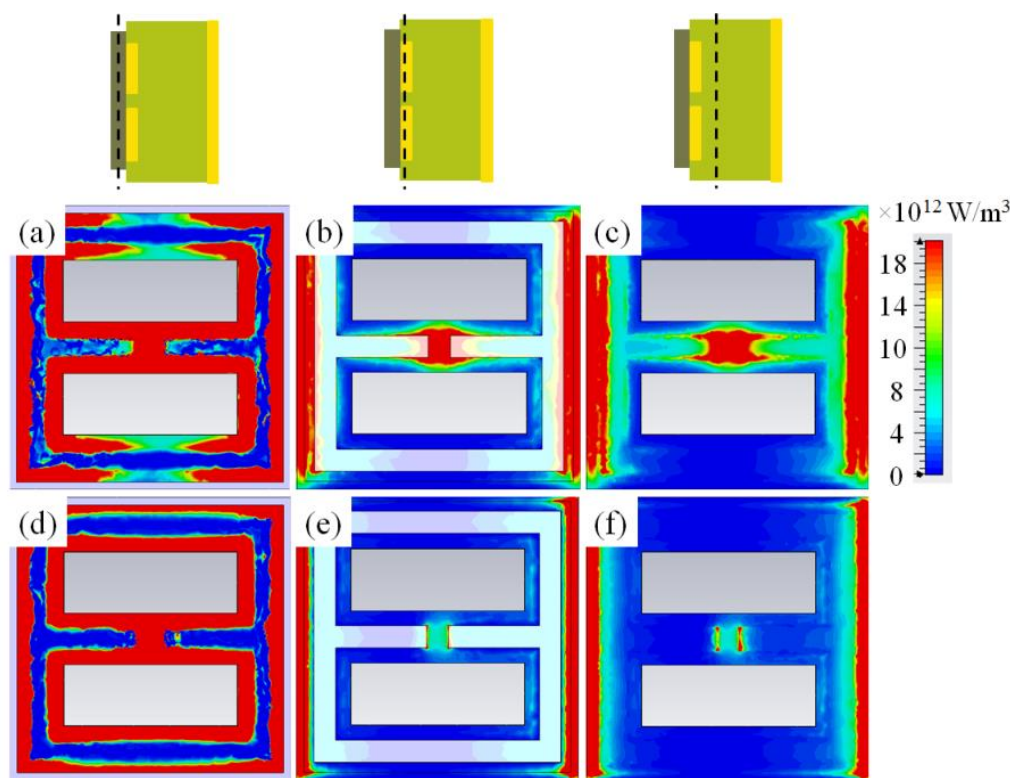

Figure 8: Simulated power loss at different slice across the MPA structure. (a) - (c) The power loss in different material when $\sigma \mathrm{GaAs}$ is $1 \mathrm{~S} / \mathrm{cm}$. (d) - (f) The power loss when $\sigma \mathrm{GaAs}$ is $150 \mathrm{~S} / \mathrm{cm}$. 